\title{
Optimal Management with Potential Regime Shifts
}

\author{
Stephen Polasky ${ }^{1}$ \\ Aart de Zeeuw ${ }^{2}$ \\ Florian Wagener ${ }^{3}$
}

June 18, 2010

\begin{abstract}
$\underline{\text { Abstract }}$
We analyze how the threat of a potential future regime shift affects optimal management. We use a simple general growth model to analyze four cases that involve combinations of stock collapse versus changes in system dynamics, and exogenous versus endogenous probabilities of regime shift. Prior work has focused on stock collapse with endogenous probabilities and reaches ambiguous conclusions about the effect of potential regime shift on optimal management. We show that all other cases yield unambiguous results. In particular, with endogenous probability of regime shift that affects system dynamics the potential for regime shift causes optimal management to become precautionary.
\end{abstract}

JEL-codes: E61, O40, Q20, C61

Keywords: optimal management, growth, renewable resources, regime shifts

\footnotetext{
${ }^{1}$ University of Minnesota, NBER, and Beijer Institute of Ecological Economics

2 TSC, Tilburg University and Beijer Institute of Ecological Economics

${ }^{3}$ CeNDEF, University of Amsterdam
} 


\title{
Optimal Management with Potential Regime Shifts
}

\begin{abstract}
$\underline{\text { Abstract }}$
We analyze how the threat of a potential future regime shift affects optimal management. We use a simple general growth model to analyze four cases that involve combinations of stock collapse versus changes in system dynamics, and exogenous versus endogenous probabilities of regime shift. Prior work has focused on stock collapse with endogenous probabilities and reaches ambiguous conclusions about the effect of potential regime shift on optimal management. We show that all other cases yield unambiguous results. In particular, with endogenous probability of regime shift that affects system dynamics the potential for regime shift causes optimal management to become precautionary.
\end{abstract}

JEL-codes: E61, O40, Q20, C61

Keywords: optimal management, growth, renewable resources, regime shifts 


\section{Introduction}

Complex dynamic systems can undergo changes in interactions between constituent components that cause a shift to different system dynamics. In ecological systems such "regime shifts" can cause changes in nutrient cycling and population dynamics of various species with consequences for the value of ecosystem services derived from the system. For example, lakes may shift between oligotrophic and eutrophic conditions with impacts on water quality, fish populations, recreation, and aesthetics (Scheffer 1997, Carpenter et al. 1999, Carpenter 2003). Terrestrial systems can shift between grasslands and woodlands with impacts on the value of grazing and other ecosystem services (Perrings and Walker 1997, Janssen et al. 2004). Coral reef systems can shift from coral dominated to algal dominated with impacts on water quality, fish populations, recreation and aesthetics (Hughes et al. 2003). At a larger scale, the global climate system may have regime shifts with potentially major consequences in several dimensions (e.g., sea level rise, agricultural production, water scarcity). Economic systems can also undergo regime shifts. Examples include sudden shifts in consumer choices ("fads") and cultural change (e.g., Bikhchandani et al. 1992, and popularized by Gladwell 2000), shifts in financial markets due to changes in investor sentiment and herd behavior (e.g., Scharfstein and Stein 1990, Banerjee 1992, Brock and Hommes 1998) or due to changes in investor information and hedging (Genotte and Leland 1990, Brock et al. 2009), and shifts in the macro-economy (e.g., Azariadis 1981, Cass and Shell 1983, Hamilton 1989, Guo et al. 2005). Once a threshold between regimes has been crossed it may be difficult to reverse the process to shift back to the original regime ("system hysteresis", Scheffer et al. 2001).

In this paper we analyze optimal management of a dynamic system with the potential for a regime shift. To fix ideas, we focus our discussion on the case of harvesting a renewable resource (e.g., a fishery) in which the growth function of the stock is dependent on the regime and where the stock level of the resource can influence the probability of a regime shift. For example, high levels of harvest can reduce fish populations that graze on plankton and increase the probability that a bleaching event or other disturbance will shift a coral reef system from coral dominated to algal dominated (Hughes et al. 2003). The shift into a new regime reduces fishery productivity and may also reduce other ecosystem services (recreation, storm protection, etc). The model we analyze, however, is more general than harvesting a renewable resource and could be used to analyze any circumstance in which management 
actions affect probabilities of regime shift, such as greenhouse gas emissions and climate regime shifts, or financial regulations and the potential for sudden shifts in investor sentiment.

Prior research in economics on optimal management with potential regime shift has focused on the case of catastrophic stock collapse. In environmental economics, this line of research began with Cropper (1976) who analyzed a model in which utility falls to zero once a threshold is crossed. ${ }^{4}$ In Cropper's model, the location of the threshold is unknown. The probability of crossing the threshold increases in the level of pollution (or resource depletion). William Reed showed how to transform the optimal management problem with a probability of crossing a threshold, which is a stochastic dynamic problem, into a deterministic problem that could be solved analytically using the Pontryagin maximum principle (Reed 1987, 1988; and see Reed and Echavarria Heras 1992 for a useful summary). In Reed's approach, the potential for collapse has an ambiguous effect on management prior to the collapse. The potential for collapse tends to increase exploitation because collapse reduces the future value of stocks so there is less incentive to maintain stocks. This effect works identically to an increase in the discount rate and occurs for the same reason that an increase in mortality risk increases an individual's discount rate. Working in the opposite direction, however, is the fact that decreased exploitation results in higher stocks and lowers the probability of collapse. We refer to actions that lessen exploitation to reduce probabilities of bad future outcome as "precaution". Combining these two effects yields an ambiguous overall result. Reed applied his approach to analyze optimal management of forests subject to fire (Reed 1984, 1987, 1989), fisheries subject to collapse (Reed 1988) and environmental pollution (Clarke and Reed 1994). Threshold models have also been applied by other researchers to climate change (e.g., Tsur and Zemel 1996, Gjerde et al. 1999, Keller et al. 2004), environmental pollution (Tsur and Zemel 1998), groundwater aquifers (Tsur and Zemel 1995), and nuclear power (Aronsson et al. 1998). Mäler et al. (2007) apply a similar model to generate a shadow price for resilience, where resilience influences the probability of regime shift. A slightly different modeling approach to thresholds was taken by Nævdal (2001, 2003, 2006) who does not include the potential for shocks so that the probability of regime shift is positive only in time periods when stocks are being depleted. Tsur and Zemel $(2006,2008)$ study the regulation of stock externalities that arise in cases of non-cooperative behavior. In all of these models, a regime shift triggers a discontinuous decline in a state variable and/or value function.

\footnotetext{
${ }^{4}$ Earlier, Kamien and Schwarz (1971) developed a model of machinery failure that is formally similar to models of environmental collapse.
} 
A different approach, and one more in line with the ecological literature, is to model a regime shift as a change in system dynamics rather than as a sudden collapse in the stock. Peterson, Carpenter and Brock (2003) consider a model with two regimes (oligotrophic and eutrophic lake system), with state equations that differ by an additive term. They show that optimal management will typically involve periodic collapse (switch from oligotrophic to eutrophic) and recovery (switch from eutrophic to oligotrophic). Brozovic and Schlenker (2008) use a similar model of regime shift to analyze the relationship between precaution and variance of uncertainty and find that the relationship is not monotonic. An increase in the variance of the stochastic component of the natural system that determines whether the threshold is crossed initially increases precaution. However, if the variance gets very large, not much can be done to prevent crossing the threshold and precaution becomes too costly compared to the small reduction in the probability that the threshold is crossed. Brock and Starrett (2003) and Mäler et al. (2003) analyze a model with a convex-concave regeneration function that captures the potential for regime shifts. These models, however, are deterministic and focus on characterizing different optimal paths under various parameter conditions.

In this paper we develop a general growth model with stochastic regime shift that can capture changes to stock levels and/or system dynamics when a regime shift occurs. We consider cases in which the probability of a regime shift is not affected by any management action (exogenous regime shift), and cases in which the probability of a regime shift is a function of management action (endogenous regime shift). The model in this paper contains one important simplification. We assume that the objective function is linear in the control variable, which generates a bang-bang control and allows for a relatively simple analytical solution.

In the case with an exogenous regime shift that results in a change in system dynamics but not an immediate change in stock level, we show that the threat of regime shift does not affect optimal management prior to any potential regime shift. With an endogenous regime shift that changes system dynamics, optimal management becomes precautionary in the sense that the potential for regime shift will cause managers to choose less intensive harvest (emissions) and maintain higher resource stocks (environmental quality). These results contrast with the prior literature that focused on stock collapse where a potential regime shift causes more intensive exploitation (in the case with exogenous regime shift) or ambiguous results (in the case with endogenous regime shift). 
In the next section we set up the optimal management model with potential regime shifts and derive results. The results are discussed in section 3 . We compare our results with prior literature and explain major differences. We summarize our findings and include a brief discussion of important extensions and open questions in section 4.

\section{Model}

We use a simple dynamic model with a linear objective function (constant price and constant marginal cost of harvest) to demonstrate results about optimal management with the potential for a regime shift. The linear objective function in the control variable simplifies the analytics. Other than this, however, the model is quite general. For concreteness and to make comparisons with prior literature easier, we interpret the model as a renewable resource model. The renewable resource can be thought of as a traditional renewable resource like a fishery or as an environmental resource like the global atmosphere. Additional harvest of fish, or increased emission of greenhouse gases, generates extra current flow benefits but leads to depletion of the resource base, which pushes the system in an undesirable direction and makes it more likely that some shock or disturbance will cause regime shift.

The objective is to maximize the present value of revenue from harvest, subject to stock dynamics

$$
\begin{aligned}
& \operatorname{Max} \int_{0}^{\infty} e^{-r t} p h(t) d t \\
& \text { s.t. } \dot{s}(t)=G(s(t))-h(t), s(0)=s_{0}, s(t) \geq 0, h(t) \geq 0 \text { for all } t \text {, }
\end{aligned}
$$

where $p>0$ denotes the constant net price, $h(t)$ is the harvest level at time $t, r>0$ is the discount rate, $s(t)$ is the resource stock at time $t$, and $G(s(t))$ is the natural growth function of the resource, which depends on the resource stock. We assume that the growth function is concave in stock and that $G(0)=0$.

At some future time $\tau$ (possibly infinite) there is a regime shift. Before time $\tau$ the growth function is given by $G_{l}(s)$, with $G_{l}(s)>0$ for $0<s<K_{l}$ and $G_{l}(s)<0$ for $s>K_{l}$. In the case where regime shift changes system dynamics, after time $\tau$ the growth function is given by $G_{2}(s)$, with $G_{2}(s)>0$ for $0<s<K_{2}$ and $G_{2}(s)<0$ for $s>K_{2}$. We assume that $G_{1}(s)$ $>G_{2}(s)$ and $G_{1}{ }^{\prime}(s)>G_{2}{ }^{\prime}(s)$, for all $s>0$, and $K_{2}<K_{1}$. Here $K_{i}, i=1,2$, can be thought of as carrying capacity, i.e. the steady-state value in the system with no harvest. The size of the stock at time $\tau$ is unchanged by the regime shift. Stock dynamics are characterized by 


$$
\dot{s}(t)=G(s(t))-h(t)=\left\{\begin{array}{l}
G_{1}(s(t))-h(t), \text { for } 0 \leq t<\tau \\
G_{2}(s(t))-h(t), \text { for } t \geq \tau
\end{array} .\right.
$$

A simple illustration of the effect of the regime shift for the case of logistic growth, $G_{i}(s)=$ $g s\left(1-s / K_{i}\right), i=1,2$, with $g=1, K_{1}=1$ and $K_{2}=0.75$, is shown in Figure 1.

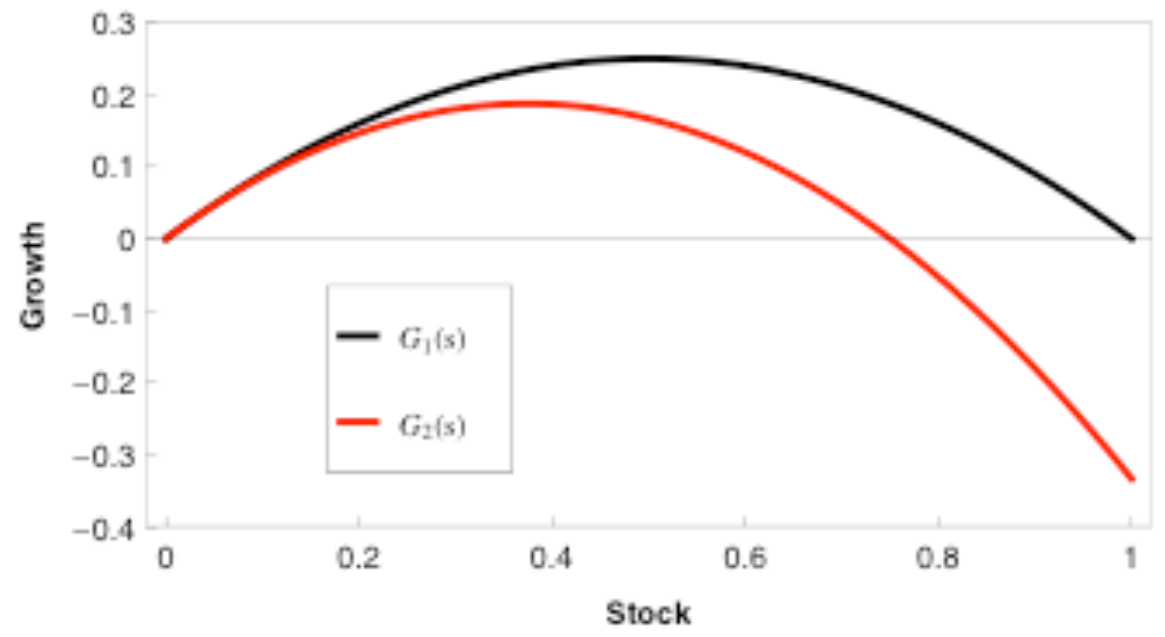

Figure 1: Illustration of a reduced growth function after a regime shift.

In the case where regime shift causes stock collapse, we have $s(t)=0$ and $G_{2}(0)=0$ for all $t \geq \tau$.

We think of the regime shift as a future event where the time until this event occurs is a stochastic variable. The standard way of modeling this is by means of a hazard rate $\lambda$. If the hazard rate is constant, the time until the event occurs is drawn from an exponential probability distribution $\lambda e^{-\lambda x}$ where a high $\lambda$ means a high probability that the event will happen soon. Alternatively, $1 / \lambda$ can be seen as an indicator of the resilience of the system, i.e., its ability to resist shocks and maintain current productivity even with disturbance (note that $1 / \lambda$ is the mean of the exponential probability distribution). We will, however, also consider hazard rates that depend on the stock, $\lambda(s)$, in order to capture the possibility that harvesting behavior that changes stock levels will affect the probability of a regime shift.

In the case where regime shift causes a shift in system dynamics, we have a standard renewable resource model with growth function $G_{2}(s)$ after the shift has occurred. The optimal harvesting policy in this second regime can be derived with a "most rapid approach path" technique (Spence and Starrett 1975). However, we shall use dynamic programming as that technique can also be used when analysing the first regime before the shift has occurred. 
Except for discounting, the problem is stationary and the Hamilton-Jacobi-Bellman equation for the current value function $V_{2}$ is given by

$$
0=\max _{h}\left\{p h-r V_{2}(s)+V_{2}^{\prime}(s)\left(G_{2}(s)-h\right)\right\} .
$$

Because this equation is linear in $h$, the optimal harvest is a bang-bang control with $h=0$ when $p<V_{2}^{\prime}(s), h$ infinite when $p>V_{2}^{\prime}(s)$, and $h$ indeterminate when $p=V_{2}^{\prime}(s)$. We first fix a maximal harvest level $h_{m}$ large enough to have the stock always decreasing for $h=h_{m}$, and determine the solution $V_{2}$ of this problem. We then take the limit $h_{m} \rightarrow \infty$ in $V_{2}$. We will construct the solution in the following way. Suppose we have a positive state $s_{2}$ below the carrying capacity $K_{2}$ with $h=0$ for $s<s_{2}$ and $h=h_{m}$ for $s>s_{2}$. If $h=0$ and $h=h_{m}$ are the optimal harvest levels, the Hamilton-Jacobi-Bellman equation (3) yields

$$
\left\{\begin{array}{l}
0=-r V_{2}(s)+V_{2}{ }^{\prime}(s) G_{2}(s), \text { for } s<s_{2}, \\
0=p h_{m}-r V_{2}(s)+V_{2}{ }^{\prime}(s)\left(G_{2}(s)-h_{m}\right), \text { for } s>s_{2} .
\end{array}\right.
$$

In the limit as $s$ approaches $s_{2}$ from below, and $s$ approaches $s_{2}$ from above, equations (4a) and (4b) can be interpreted as two equations in the two unknowns $V_{2}\left(s_{2}\right)$ and $V_{2}{ }^{\prime}(s)$ (requiring the function $V_{2}$ to be continuously differentiable) which leads to

$$
V_{2}^{\prime}\left(s_{2}\right)=p, \quad V_{2}\left(s_{2}\right)=\frac{p G_{2}\left(s_{2}\right)}{r} .
$$

Differentiation and algebraic manipulation of equations (4a) and (4b) yields

$$
\left\{\begin{array}{l}
V_{2}^{\prime \prime}(s)=\left(r-G_{2}{ }^{\prime}(s)\right) \frac{r V_{2}(s)}{\left(G_{2}(s)\right)^{2}}, \text { for } s<s_{2}, \\
V_{2}^{\prime \prime}(s)=\left(r-G_{2}{ }^{\prime}(s)\right) \frac{r V_{2}(s)-p h_{m}}{\left(G_{2}(s)-h_{m}\right)^{2}}, \text { for } s>s_{2} .
\end{array}\right.
$$

Assuming that $G_{2}{ }^{\prime}(0)>r$, the state $s_{2}$ is determined by

$$
G_{2}{ }^{\prime}\left(s_{2}\right)=r \text {. }
$$

It is clear from equation (1) that $0<V_{2}(s)<p h_{m} / r$. Therefore, it follows from equation (6) and the concavity of $G_{2}(s)$ that $V_{2}^{\prime \prime}(s)$ is negative for $s<s_{2}$ and negative for $s>s_{2}$, so that $V_{2}^{\prime}(s)$ is decreasing throughout. With equation (5), this implies that $V_{2}^{\prime}(s)>p$ for $s<s_{2}$ and $V_{2}^{\prime}(s)<p$ for $s>s_{2}$, so that $h=0$ and $h=h_{m}$ are indeed the optimal harvest levels for $s<s_{2}$ and $s>s_{2}$, respectively. All the conditions of the Hamilton-Jacobi-Bellman equation (3) are satisfied. The structure of the optimal solution in the second regime is clear now. It consists of the steady-state path $s=s_{2}$, where $s_{2}$ is determined by equation (7), with $h=G_{2}\left(s_{2}\right)$, either 
preceded by a path with $h=0$ (if we start at a stock $s$ below $s_{2}$ ) or by a path with $h=h_{m}$ (if we start at a stock $s$ above $s_{2}$ ).

Equation (7) is the standard "golden rule" of growth. The value function $V_{2}$ is explicitly given by

$$
V_{2}(s)=\left\{\begin{array}{l}
e^{-r t_{0}(s)} \frac{p G_{2}\left(s_{2}\right)}{r}, \text { for } s<s_{2} \\
e^{-r t_{m}(s)} \frac{p G_{2}\left(s_{2}\right)}{r}+\left(1-e^{-r t_{m}(s)}\right) \frac{p h_{m}}{r}, \text { for } s>s_{2}
\end{array},\right.
$$

where $t_{0}(s)$ and $t_{m}(s)$ can be interpreted as the times needed to reach $s_{2}$ from $s$ with $h=0$ and $h=h_{m}$, respectively. These satisfy $t_{0}{ }^{\prime}(s)=-1 / G_{2}(s)$ and $t_{m}{ }^{\prime}(s)=-1 /\left(h_{m}-G_{2}(s)\right)$. We now take the limit of the value function $V_{2}$ for $s>s_{2}$, given by equation (8), for $h_{m} \rightarrow \infty$. It is clear that $t_{m}(s) \rightarrow 0$. Furthermore, since $\lim _{x \rightarrow 0}\left(1-e^{-x}\right) / x=1$, we have

$$
\lim _{h_{m} \rightarrow \infty} \frac{1-e^{-r t_{m}(s)}}{r t_{m}(s)} t_{m}(s) p h_{m}=\lim _{h_{m} \rightarrow \infty} p \int_{s_{2}}^{s} \frac{h_{m}}{h_{m}-G_{2}(x)} d x=p \int_{s_{2}}^{s} d x=p\left(s-s_{2}\right) .
$$

In the limit, the value function $V_{2}(s)$ for $s>s_{2}$ takes the form

$$
V_{2}(s)=p\left(s-s_{2}\right)+\frac{p G_{2}\left(s_{2}\right)}{r}, \text { for } s>s_{2} .
$$

In the limit for $h_{m} \rightarrow \infty$, the difference $\left(s-s_{2}\right)$ is harvested instantaneously and sold at price $p$.

Deriving the value function in the case where a regime shift causes a stock collapse is trivial since $s(t)=0$, so that $h(t)=0$ for all $t \geq \tau$. Therefore, we have $V_{2}(s)=0$ for all $s$.

We now consider the first regime. Harvest levels $h$ must maximize the expected present value of net revenue

$$
\begin{aligned}
& \operatorname{Max} E\left\{\int_{0}^{\tau} e^{-r t} p h(t) d t+e^{-r \tau} V_{2}(s(\tau))\right\} \\
& \text { s.t. } \dot{s}(t)=G_{1}(s(t))-h(t), s(0)=s_{0}, s(t) \geq 0, h(t) \geq 0 \text { for all } t,
\end{aligned}
$$

where $\tau$ is a stochastic variable. For a constant hazard rate $\lambda$, with an exponential probability distribution for the point in time $\tau$, deriving the optimal solution using a Pontryagin approach is straightforward (see the appendix). However, we want to consider the possibility that the hazard rate $\lambda$ is not constant and that it depends on the stock $s$. This can be solved with a Pontryagin approach as well (see the appendix) but we prefer to develop the HamiltonJacobi-Bellman equation for the value function in the first regime, directly using the hazard rate, because the analysis is more transparent.

Starting at time $t$ with stock $s$ we can approximate the probability of a regime shift in a small time period $\Delta t$ by $\lambda(s(t)) \Delta t$, which is in fact the basic definition of the hazard rate $\lambda$. 
The value function, $W_{l}(s, t)$, is the maximal expected value of the objective function at time $t$ for stock $s$ and can therefore be written as

$$
\begin{aligned}
W_{1}(s, t)=\max _{h}\left\{\int_{t}^{t+\Delta t} e^{-r x} p h(x) d x\right. \\
\left.\quad+(1-\lambda(s) \Delta t) W_{1}(s+\Delta s, t+\Delta t)+\lambda(s) \Delta t V_{2}(s+\Delta s)\right\}
\end{aligned}
$$

We use the symbol $W$ because we want to use the symbol $V$ when we eliminate the factor $e^{-r t}$ from the resulting Hamilton-Jacobi-Bellman equation below. By approximating the integral and moving the left-hand side of equation (12) to the right-hand side and dividing by $\Delta t$, we get

$$
\begin{gathered}
0=\max _{h}\left\{e^{-r t} p h+\lambda(s) V_{2}(s+\Delta s)-\lambda(s) W_{1}(s+\Delta s, t+\Delta t)\right. \\
\left.+\frac{W_{1}(s+\Delta s, t+\Delta t)-W_{1}(s, t)}{\Delta t}\right\}
\end{gathered} .
$$

Taking the limit of equation (13) for $\Delta t \rightarrow 0$ yields

$$
0=\max _{h}\left\{e^{-r t} p h+\lambda(s) V_{2}(s)-\lambda(s) W_{1}(s, t)+W_{1 s}(s, t)\left(G_{1}(s)-h\right)+W_{1 t}(s, t)\right\} .
$$

Except for discounting, the problem is stationary again. By defining $V_{l}(s)=e^{r t} W_{l}(s, t)$, the Hamilton-Jacobi-Bellman equation for the first regime becomes

$$
0=\max _{h}\left\{p h+\lambda(s)\left(V_{2}(s)-V_{1}(s)\right)-r V_{1}(s)+V_{1}^{\prime}(s)\left(G_{1}(s)-h\right)\right\} .
$$

The structure of equation (15) is the same as the structure of equation (3). The optimal harvest in the first regime is a bang-bang control as well, with $h=0$ when $p<V_{1}^{\prime}(s), h$ infinite when $p>V_{1}^{\prime}(s)$, and $h$ indeterminate when $p=V_{1}^{\prime}(s)$. Again, we first impose the restriction $0 \leq h \leq h_{m}$. As before we search for a positive state $s_{l}$, below the carrying capacity $K_{1}$, with $h=0$ for $s<s_{1}$ and $h=h_{m}$ for $s>s_{1}$, so that all the conditions of the HamiltonJacobi-Bellman equation (15) are satisfied. If $h=0$ and $h=h_{m}$ are the optimal harvest levels, equation (15) yields

$$
\left\{\begin{array}{l}
0=\lambda(s) V_{2}(s)-(r+\lambda(s)) V_{1}(s)+V_{1}^{\prime}(s) G_{1}(s), \text { for } s<s_{1} \\
0=p h_{m}+\lambda(s) V_{2}(s)-(r+\lambda(s)) V_{1}(s)+V_{1}^{\prime}(s)\left(G_{1}(s)-h_{m}\right), \text { for } s>s_{1}
\end{array}\right.
$$

In the limit as $s$ approaches $s_{1}$ from below, and $s$ approaches $s_{1}$ from above, equations (16a) and (16b) yield two equations in the two unknowns $V_{l}\left(s_{l}\right)$ and $V_{l}{ }^{\prime}\left(s_{l}\right)$ (requiring the function $V_{l}$ to be continuously differentiable) which leads to

$$
V_{1}^{\prime}\left(s_{1}\right)=p, \quad V_{1}\left(s_{1}\right)=\frac{p G_{1}\left(s_{1}\right)+\lambda\left(s_{1}\right) V_{2}\left(s_{1}\right)}{r+\lambda\left(s_{1}\right)} .
$$

Differentiation of equations (16a) and (16b) yields 


$$
\left\{\begin{array}{l}
G_{1}(s) V_{1}^{\prime \prime}(s)=f(s), \text { for } s<s_{1}, \\
\left(G_{1}(s)-h_{m}\right) V_{1}^{\prime \prime}(s)=f(s), \text { for } s>s_{1},
\end{array}\right.
$$

where

$$
f(s)=\left(r+\lambda(s)-G_{1}{ }^{\prime}(s)\right) V_{1}{ }^{\prime}(s)-\lambda(s) V_{2}{ }^{\prime}(s)+\lambda^{\prime}(s)\left(V_{1}(s)-V_{2}(s)\right) .
$$

In order for $h=0$ and $h=h_{m}$ to be the optimal harvest levels, we need that $V_{1}^{\prime}(s)>p$ for $s<$ $s_{1}$ and $V_{1}^{\prime}(s)<p$ for $s>s_{1}$. As a consequence, with equation (17), we need the left limit and the right limit of $V_{1}^{\prime \prime}(s)$ at $s=s_{1}$ to be less than or equal to 0 . It follows from equation (18) that this is equivalent to $f(s) \leq 0$ for $s<s_{1}$ and $f(s) \geq 0$ for $s>s_{1}$, so that the state $s_{1}$ must satisfy $f\left(s_{1}\right)=0$ or, using equations (19) and (17),

$$
G_{1}{ }^{\prime}\left(s_{1}\right)=r+\lambda\left(s_{1}\right)\left[1-\frac{V_{2}{ }^{\prime}\left(s_{1}\right)}{p}\right]+\frac{\lambda^{\prime}\left(s_{1}\right)}{r+\lambda\left(s_{1}\right)}\left[G_{1}\left(s_{1}\right)-\frac{r}{p} V_{2}\left(s_{1}\right)\right] .
$$

The difficulty here is that we cannot generally show that $f(s)<0$ for $s<s_{1}$ and $f(s)>0$ for $s$ $>s_{1}$, as we had in the analysis of the second regime. It is reasonable to assume that $\lambda^{\prime}(s)<0$, because the resilience $1 / \lambda(s)$ should increase if the stock $s$ increases, and that $\lambda^{\prime}\left(K_{1}\right)=0$. Furthermore, we assume that $G_{1}{ }^{\prime}(0)>r+\lambda(0)$. This guarantees that $f(0)<0$ and $f\left(K_{l}\right)>0$, so that the function $f$ is zero and switches sign from negative to positive somewhere on the interval $\left(0, K_{l}\right)$. Note, however, that the function $f$ may have multiple zeros. If $f$ switches from positive to negative, the solution of equation (20) separates areas where $h=h_{m}$ to the left and $h=0$ to the right. These points are not stable. We cannot rule out the existence of more than one stable steady state, however, so that the optimal solution may depend on the initial state. If there is an interval of singular states, instead of an isolated one, we need to have $V_{1}^{\prime}(s)=p$ on that interval. It follows from equation (15) that equation (17) and thus equation (20) have to hold on that interval. This is generally not possible. In what follows, we will focus on the smallest steady state $s_{1}$, satisfying equation (20) with $f(s)$ $<0$ for $s<s_{1}$ and $f(s)>0$ for $s>s_{1}$ in some neighborhood of $s_{1}$. The function $V_{1}$ satisfying the differential equation (16), with initial condition (17), solves the Hamilton-Jacobi-Bellman equation (15) in this neighborhood. It follows that the optimal solution here consists of a steady-state path $s=s_{l}$, with $h=G_{l}(s)$, either preceded by a path with $h=0$ (if we start at a stock $s$ below $s_{1}$ ) or by a path with $h=h_{m}$ (if we start at a stock $s$ above $s_{1}$ ). 
Equation (20) is the "golden rule" of growth when there is the possibility of a regime shift. Our results will follow directly from this golden rule so that we do not give an explicit expression for the value function $V_{l}$ where $h_{m} \rightarrow \infty .^{5}$

\section{Results}

We can use the results of the analysis summarized in equation (20) to provide a characterization of the effects of regime shift on optimal management. We distinguish four cases: a) exogenous regime shift with stock collapse, b) endogenous regime shift with stock collapse, c) exogenous regime shift with changed system dynamics, and d) endogenous regime shift with changed system dynamics.

\section{Case 1: Exogenous regime shift with stock collapse}

With a constant hazard rate $\lambda$ and a stock collapse, we have that $\lambda^{\prime}(s)=0$ and $V_{2}=0$ so that the condition for the steady-state stock prior to a regime shift (as shown in equation (20)) becomes

$$
G_{1}{ }^{\prime}\left(s_{1}\right)=r+\lambda .
$$

This result shows that potential future regime shift increases the discount rate leading to a lower steady-state stock than without the possibility of collapse.

\section{Case 2: Endogenous regime shift with stock collapse}

If the hazard rate $\lambda$ depends on the stock $s$, the condition for the steady-state stock prior to a regime shift with potential total stock collapse $V_{2}=0$ becomes

$$
G_{1}{ }^{\prime}\left(s_{1}\right)=r+\lambda\left(s_{1}\right)+\frac{\lambda^{\prime}\left(s_{1}\right) G_{1}\left(s_{1}\right)}{r+\lambda\left(s_{1}\right)} .
$$

In this case we have two effects and the net effect is ambiguous (note that $\lambda^{\prime}(s)<0$ ). The effect shown in Case 1 above (the addition of the term $\lambda(s)$ ) will tend to increase the intensity of harvest and decrease the steady-state stock. However, the desire to avoid a regime shift will tend to decrease the intensity of harvest and increase the steady-state stock because the final term $\frac{\lambda^{\prime}\left(s_{1}\right) G_{1}\left(s_{1}\right)}{r+\lambda\left(s_{1}\right)}$ in equation (22) is negative. The overall effect is ambiguous. The effect of the potential regime shift on steady-state stock will depend on

\footnotetext{
${ }^{5}$ The expressions are available from the authors upon request.
} 
which effect dominates. The previous literature has focused on this case (e.g., Reed 1988, Clarke and Reed 1994, Tsur and Zemel 1996).

\section{Case 3: Exogenous regime shift with changed system dynamics}

If the hazard rate $\lambda$ is constant and the regime shift causes a shift in system dynamics we have that $\lambda^{\prime}(s)=0$ and $V_{2}^{\prime}\left(s_{1}\right)=p$ so that the condition for the steady-state stock prior to a regime shift becomes

$$
G_{1}{ }^{\prime}\left(s_{1}\right)=r .
$$

The steady-state stock in this case is the same as without the possibility of a regime shift. An exogenous probability of regime shift that causes a change in system dynamics, but not an immediate change in stock levels, will not change optimal management. Note that $s_{l}$ is larger than $s_{2}$ (determined by equation (7)) because of the conditions on the growth functions $G_{1}$ and $G_{2}$ in section 2. If the regime shift occurs, management will adjust to the new situation, with a lower steady-state value. However, prior to regime shift it is optimal to manage according to the current (not future) conditions.

\section{Case 4: Endogenous regime shift with changed system dynamics}

If the hazard rate $\lambda$ depends on the stock $s$ and a regime shift causes a shift in system dynamics, the condition for the steady-state stock prior to a regime shift becomes

$$
G_{1}^{\prime}\left(s_{1}\right)=r+\frac{\lambda^{\prime}\left(s_{1}\right)}{r+\lambda\left(s_{1}\right)}\left[G_{1}\left(s_{1}\right)-\frac{r}{p} V_{2}\left(s_{1}\right)\right]
$$

which can be rewritten as

$$
G_{1}^{\prime}\left(s_{1}\right)=r+\frac{\lambda^{\prime}\left(s_{1}\right)}{r+\lambda\left(s_{1}\right)} \frac{r}{p}\left[\frac{p G_{1}\left(s_{1}\right)}{r}-V_{2}\left(s_{1}\right)\right] .
$$

The term between brackets is positive which can be seen as follows. With initial state $s_{1}$, the objective in (11) is maximized by $h=G_{l}\left(s_{l}\right)$. It follows that

$$
V_{1}\left(s_{1}\right)=E\left\{\int_{0}^{\tau} e^{-r t} p G_{1}\left(s_{1}\right) d t+e^{-r \tau} V_{2}\left(s_{1}\right)\right\}=\left(1-E e^{-r \tau}\right)\left[\frac{p G_{1}\left(s_{1}\right)}{r}-V_{2}(s)\right]+V_{2}\left(s_{1}\right) .
$$

$V_{l}\left(s_{1}\right)$ must be larger than $V_{2}\left(s_{1}\right)$ because the maximizing $h=G_{l}\left(s_{1}\right)$ must give a larger value than implementing the optimal harvesting policy for the second regime from the beginning. It follows that the term between brackets in equation (25) is positive. Furthermore, because $\lambda^{\prime}\left(s_{1}\right)<0$, it is clear now that $G_{1}{ }^{\prime}\left(s_{1}\right)<r$, so that optimal management is precautionary in this case: the potential for a regime shift will decrease the harvest rate and increase the 
steady-state stock level. How much the steady-state stock will increase under precautionary optimal management depends on the difference between the growth functions $\left(G_{1}(s)\right.$ and $\left.G_{2}(s)\right)$, on the probability characteristics of the regime shift $(\lambda(s))$, and on the discount rate $(r)$.

\section{Discussion}

As we demonstrated above, for the case when regime shift causes a shift in system dynamics and when management actions influence the probability of a shift from a desirable to an undesirable regime, optimal management involves precautionary actions that reduce the probability of regime shift. This result, where uncertainty regarding potentially harmful future consequences leads to actions that reduce potential risks, accords with much recent writing in environmental and resource management on the "Precautionary Principle" (e.g. Lauck et al. 1998, Raffensperger and Tickner 1999). To date, the Precautionary Principle has lacked rigorous justification except under rather limited conditions (Gollier et al. 2000). However, with an exogenous probability of regime shift (i.e., when management actions do not affect the probability of regime shift) and where the regime shift affects system dynamics but does not cause stock collapse, optimal management prior to regime shift is unaffected by the potential for regime shift. Once a regime shift has occurred, management will be adjusted to fit the new conditions. Any change in management prior to the regime shift involves a loss of profit from failing to satisfy the golden rule of growth in the initial regime.

These results contrast with prior results in the literature that show that the potential for a catastrophic collapse in stock will cause either an increase in exploitation (which is the opposite of precautionary action) or an ambiguous effect. In the models of catastrophic collapse of a renewable resource such as a fishery, the collapse causes the stock of a renewable resource to fall, with either no future harvest or an eventual recovery to pre-crash conditions. In either event, the collapse causes the destruction of a valuable asset. Knowing that there is some potential for asset destruction, a manager will be more aggressive in harvesting the resource in order to gain profits prior to potential destruction. So, for example, the risk of a forest fire that would destroy timber assets gives an incentive for a landowner to harvest timber sooner than if there were no risk of fire (Reed 1984). This stock effect works to increase the manager's discount rate in the same way that an increase in risk of mortality increases the discount rate for an individual. The increase in the discount rate shifts optimal management towards immediate consumption and away from savings and investment.

We summarize the differences between our results where regime shift leads to changes in system dynamics and the prior literature on stock collapse in Table 1. Regime 
shifts with changes in system dynamics (but no stock effect) do not cause a change in optimal management by themselves (Case III). Only if management actions lead to changes in the probability of a regime shift (via changes in the state of the system) will the potential for regime shift lead to changes in optimal management (Case IV). In this case, optimal management is to become precautionary in the sense that a threat of future regime shift will cause managers to reduce current harvests and increase the stock of the resource. For climate change, this would mean that consideration of the potential of future regime shifts should cause a decrease in current emissions of greenhouse gases. In contrast, the potential for stock collapse itself causes a shift in optimal management towards more aggressive exploitation (Case I). With endogenous probabilities, management tends to become more precautionary, but whether this is sufficient to override the stock effect is ambiguous (Case II).

Table 1: Comparison of models and results

\begin{tabular}{|l|l|l|}
\hline & $\begin{array}{l}\text { Exogenous Probability of } \\
\text { Regime Shift }\end{array}$ & $\begin{array}{l}\text { Endogenous Probability of } \\
\text { Regime Shift }\end{array}$ \\
\hline Stock Effect & $\begin{array}{l}\text { Case I } \\
\text { Increased exploitation }\end{array}$ & $\begin{array}{l}\text { Case II } \\
\text { Ambiguous result }\end{array}$ \\
\hline System Dynamics Effect & Case III & $\begin{array}{l}\text { Case IV } \\
\text { Decreased exploitation }\end{array}$ \\
\hline
\end{tabular}

We have assumed that if the regime shift occurs, it is a once and for all shift. In reality, however, it is likely that there will be potential for the regime to shift back again to the original regime or for it to shift between a number of different regimes. Analyzing these possibilities implies a more complicated analysis with a series of nested Hamilton-JacobiBellman equations with value functions characterizing the different regimes. Doing so will change the results, of course, but not the logic that we find in our simple model above with only one potential regime shift. Another potentially fruitful avenue for future research is to extend the model of uncertainty. Using a hazard rate is a very convenient approach and it captures many important features of the problem. This approach, however, does not allow for endogenous learning about probabilities of regime shift with additional experience or with active experimentation. Incorporating the option to learn would be an interesting extension of the approach. Finally, our model assumes constant prices and abstracts from costs that change with harvest or stock levels. Having a non-linear objective function in the control 
variable would make the model more difficult to solve. In principle such a model could be solved, at least numerically. We leave such extensions for further research.

\section{$\underline{\text { References }}$}

Arnol'd, V.I. 1998. Geometrical methods in the theory of ordinary differential equations.

Springer, New York. Second Edition.

Aronsson, T., K. Backlund and K.-G. Löfgren. 1998. "Nuclear power, externalities and non-standard Pigouvian taxes: a dynamic analysis under uncertainty." Environmental and Resource Economics 11(2): 177-195.

Azariadis, C. 1981. "Self-fulfilling prophesies.” Journal of Economic Theory 25: 380-396.

Banerjee, A.V. 1992. “A simple model of herd behavior." Quarterly Journal of Economics 107(3): 797-817.

Bikhchandani, S., D. Hirschleifer and I. Welch. 1992. "A theory of fads, fashion, custom and cultural change as information cascades." Journal of Political Economy 100(5): 9921026.

Brock, W.A. and C.H. Hommes. 1998. "Heterogeneous beliefs and routes to chaos in a simple asset pricing model." Journal of Economic Dynamics \& Control 22(8/9): 1235-1274.

Brock, W.A., C.H. Hommes and F.O.O. Wagener. 2009. "More hedging instruments may destabilize markets." Journal of Economic Dynamics \& Control 33(11): 1912-1928.

Brock, W.A. and D. Starrett. 2003. "Managing systems with non-convex positive feedback." Environmental and Resource Economics 26(4): 575-602.

Brozovic, N. and W. Schlenker. 2008. "Optimal management of an ecosystem with an unknown threshold." http://ssrn.com/abstract=990613. 
Carpenter, S.R. 2003. Regime Shifts in Lake Ecosystems: Pattern and Variation. Oldendorf: International Ecology Institute.

Carpenter, S.R., D. Ludwig, and W.A. Brock. 1999. "Management and eutrophication for lakes subject to potentially irreversible change." Ecological Applications 9(3): 751-771.

Cass, D. and K. Shell. 1983. “Do sunspots matter?” Journal of Political Economy 91(2): 193-227.

Clarke, H.R. and W. J. Reed. 1994. "Consumption/pollution tradeoffs in an environment vulnerable to pollution-related catastrophic collapse." Journal of Economic Dynamics \& Control 18(5): 991-1010.

Cropper, M.L. 1976. "Regulating activities with catastrophic environmental effects." Journal of Environmental Economics and Management 3(1): 1-15.

Gennotte, G., and H. Leland. 1990. "Market liquidity, hedging, and crashes." American Economic Review 80(5): 999-1021.

Gjerde J., S. Grepperud and S. Kverndokk. 1999. "Optimal climate policy under the possibility of a catastrophe." Resource and Energy Economics 21(3/4): 289-317.

Gladwell, M. 2000. The tipping point: how little things can make a big difference. Little, Brown and Company.

Gollier, C., B. Jullien and N. Treich. 2000. "Scientific progress and irreversibility: an economic interpretation of the 'Precautionary Principle'." Journal of Public Economics 75(2): 229-253.

Grass, D., J.P. Caulkins, G. Feichtinger, G. Tragler and D.A. Behrens. 2008. Optimal control of nonlinear processes. Springer, Berlin.

Guo, X., J. Miao and E. Morellec. 2005. "Irreversible investment with regime shifts." Journal of Economic Theory 122(1): 37-59. 
Hamilton, J. 1989. "A new approach to the economic analysis of nonstationary time series and the business cycle." Econometrica 57(2): 357-384.

Hughes, T.P., A.H. Baird, D.R. Bellwood, M. Card, S.R. Connolly, C. Folke, R. Grosberg, O. Hoegh-Guldberg, J.B.C. Jackson, J. Kleypas, J.M. Lough, P. Marshall, M. Nyström, S.R. Palumbi, J.M. Pandolfi, B. Rosen and J. Roughgarden. 2003. "Climate change, human impacts, and the resilience of coral reefs." Science 301: 929-933.

Janssen, M.A., J.M. Anderies and B.H. Walker. 2004. "Robust strategies for managing rangelands with multiple stable attractors." Journal of Environmental Economics and Management 47(1): 140-162.

Kamien, M. and N. Schwartz. 1971. "Optimal maintenance and sale age for a machine subject to failure." Management Science 17(8): 495-504.

Keller, K., B.M. Bolker and D.F. Bradford. 2004. "Uncertain climate thresholds and economic optimal growth." Journal of Environmental Economics and Management 48(1): $723-741$.

Lauck, T., C.W. Clark, M. Mangel and G.R. Munro. 1998. "Implementing the Precautionary Principle in fisheries management through marine reserves." Ecological Applications 8: S72-S78.

Mäler, K.-G., A. Xepapadeas and A. de Zeeuw. 2003. "The economics of shallow lakes." Environmental and Resource Economics 26(4): 603-624.

Mäler, K.-G., C.-Z. Li and G. Destouni. 2007. "Pricing resilience in a dynamic economyenvironment system: A capital-theoretic approach." Beijer Discussion Paper 208.

Nævdal, E. 2001. "Optimal regulation of eutrophying lakes, fjords and rivers." American Journal of Agricultural Economics 83(4): 972-984. 
Nævdal, E. 2003. "Optimal regulation of natural resources in the presence of irreversible threshold effects." Natural Resource Modeling 16(3): 305-333.

Nævdal, E. 2006. "Dynamic optimization in the presence of threshold effects when the location of the threshold is uncertain with an application to a possible disintegration of the western Antarctic ice sheet." Journal of Economic Dynamics \& Control 30(7): 1131-1158.

Perrings, C. and B. Walker. 1997. "Biodiversity, resilience and the control of ecologicaleconomic systems: the case of fire-driven rangelands." Ecological Economics 22(1): 73-83.

Peterson, G.D., S.R. Carpenter, and W.A. Brock. 2003. "Uncertainty and the management of multistate ecosystems: An apparently rational route to collapse." Ecology 84(6): 14031411.

Raffensperger, C. and J. Tickner (Eds.). 1999. Protecting public health and the environment: Implementing the Precautionary Principle. Washington, DC: Island Press.

Reed, W.J. 1984. "The effects of the risk of fire on the optimal roatation of a forest." Journal of Environmental Economics and Management 11(2):180-190.

Reed, W.J. 1987. "Protecting a forest against fire: Optimal protection patterns and harvest policies." Natural Resource Modeling 2: 23-54.

Reed, W.J. 1988. "Optimal harvesting of a fishery subject to random catastrophic collapse." IMA Journal of Mathematics Applied in Medicine \& Biology 5(3): 215-235.

Reed, W.J. 1989. "Optimal investment in the protection of a vulnerable biological resource." Natural Resource Modeling 3: 463-480.

Reed, W.J. and H. Echavarria Heras. 1992. "The conservation and exploitation of vulnerable resources." Bulletin of Mathematical Biology 54(2-3): 185-207.

Scharfstein, D.S. and J.C. Stein. 1990. "Herd behavior and investment." American Economic Review 80(3): 465-479. 
Scheffer, M. 1997. The ecology of shallow lakes. London: Chapman and Hall.

Scheffer, M., S. R. Carpenter, J. A. Foley, C. Folke and B.Walker. 2001. "Catastrophic shifts in ecosystems." Nature 413: 591-596.

Spence, M. and D. Starrett. 1975. "Most rapid approach paths in accumulation problems." International Economic Review 16(2): 388-403.

Tsur, Y. and A. Zemel. 1995. "Uncertainty and irreversibility in groundwater management." Journal of Environmental Economics and Management 29(2): 149-161.

Tsur, Y. and A. Zemel. 1996. "Accounting for global warming risks: resource management under event uncertainty." Journal of Economic Dynamics \& Control 20(6/7): 1289-1305.

Tsur, Y. and A. Zemel. 1998. "Pollution control in an uncertain environment." Journal of Economic Dynamics \& Control 22(6): 967-975.

Tsur, Y. and A. Zemel. 2006. "Welfare measurement under threats of environmental catastrophes." Journal of Environmental Economics and Management 52(1): 421-429.

Tsur, Y., and A. Zemel. 2008. "Regulating environmental threats." Environmental and Resource Economics 39(3): 297-310. 


\section{Appendix 1}

a) Constant hazard rate

In the first regime with carrying capacity $K_{l}$, starting from stock levels that are higher than $s_{2}$, harvest levels $h$ must maximize the expected value of the revenue

$$
\int_{0}^{\tau} e^{-r t} p h(t) d t+e^{-r \tau} V_{2}(s(\tau))
$$

subject to stock dynamics with growth function $G_{l}$. For a constant hazard rate $\lambda$ we can easily write this expected value as follows:

$$
\int_{0}^{\infty} \lambda e^{-\lambda \tau}\left\{\int_{0}^{\tau} e^{-r t} p h(t) d t+e^{-r \tau} V_{2}(s(\tau))\right\} d \tau
$$

or, by changing the order of integration,

$$
\int_{0}^{\infty}\left(\int_{t}^{\infty} \lambda e^{-\lambda \tau} d \tau\right) e^{-r t} p h(t) d t+\int_{0}^{\infty} \lambda e^{-(r+\lambda) \tau} V_{2}(s(\tau)) d \tau,
$$

which leads to

$$
\int_{0}^{\infty} e^{-(r+\lambda) t}\left\{p h(t)+\lambda V_{2}(s(t))\right\} d t
$$

The current value Pontryagin function for this problem reads as

$$
P(s, \mu, h)=p h+\lambda V_{2}(s)+\mu\left(G_{1}(s)-h\right) .
$$

Maximizing with respect to the harvest $h$ yields that $h=0$, if $p<\mu, h=\infty$, if $p>\mu$, and $h$ is singular, if $p=\mu$. If the optimal harvest is singular on a time interval, we have there the condition $\mu(t)=p$ for the co-state $\mu$. From the co-state equation

$$
\dot{\mu}(t)=(r+\lambda) \mu(t)-\lambda V_{2}{ }^{\prime}(s(t))-\mu(t) G_{1}{ }^{\prime}(s(t))
$$

it follows that on such an interval

$$
G_{1}{ }^{\prime}(s)=r+\lambda-\lambda \frac{V_{2}{ }^{\prime}(s)}{p}
$$

for a constant $s$ with $h=G_{l}(s)$. Case 1 and case 3 follow immediately.

b) Variable hazard rate

With the hazard function $\lambda$ given by

$$
\lambda(t)=\lim _{\Delta t \rightarrow 0} \frac{P(\tau \in\{t, t+\Delta t) \mid \tau \notin\{0, t\})}{\Delta t}
$$

the probability $S$ that no regime shift has occurred up to time $t$ is given by 


$$
\begin{gathered}
S(t)=\lim _{\Delta t \rightarrow 0} \prod_{i}(1-\lambda(i \Delta t) \Delta t)=\lim _{\Delta t \rightarrow 0} \exp \left\{\sum_{i} \ln (1-\lambda(i \Delta t) \Delta t)\right\}= \\
=\lim _{\Delta t \rightarrow 0} \exp \left\{-\sum_{i} \lambda(i \Delta t) \Delta t\right\}=\exp \left\{-\int_{0}^{t} \lambda(x) d x\right\}
\end{gathered}
$$

so that the cumulative distribution function $S^{c}$ for the time $\tau$ of the regime shift becomes

$$
S^{c}(t)=P(\tau<t)=1-S(t)=1-e^{-\int_{0}^{t} \lambda(x) d x} .
$$

This yields as expected value of the revenue

$$
\int_{0}^{\infty}\left\{\int_{0}^{\tau} e^{-r t} p h(t) d t+e^{-r \tau} V_{2}(s(\tau))\right\} d S^{c}(\tau)
$$

or, by changing the order of integration,

$$
\int_{0}^{\infty}\left(\int_{t}^{\infty} d S^{c}(\tau)\right) e^{-r t} p h(t) d t+\int_{0}^{\infty} e^{-r \tau} V_{2}(s(\tau)) d S^{c}(\tau),
$$

or

$$
\int_{0}^{\infty} e^{-\int_{0}^{t} \lambda(x) d x} e^{-r t} p h(t) d t+\int_{0}^{\infty} e^{-r \tau} V_{2}(s(\tau)) \lambda(\tau) e^{-\int_{0}^{\tau} \lambda(x) d x} d \tau,
$$

which leads to

$$
\int_{0}^{\infty} e^{-\int_{0}^{(}(r+\lambda(x)) d x}\left\{p h(t)+\lambda(t) V_{2}(s(t))\right\} d t .
$$

It is convenient to introduce a second state variable $q$ by

$$
q(t)=\int_{0}^{t} \lambda(x) d x, \dot{q}(t)=\lambda(t), q(0)=0 .
$$

Assuming that the hazard rate $\lambda$ is a function of the stock $s$, the current value Pontryagin function for this problem reads as

$$
P(s, q, \mu, \sigma, h)=e^{-q}\left(p h+\lambda(s) V_{2}(s)\right)+\mu\left(G_{1}(s)-h\right)+\sigma \lambda(s) .
$$

Maximizing with respect to the harvest $h$ yields that $h=0$, if $e^{-q} p<\mu, h=\infty$, if $e^{-q} p>\mu$, and $h$ is singular, if $e^{-q} p=\mu$. If the optimal harvest is singular on a time interval, we have there the condition $\mu(t)=e^{-q(t)} p$ for the co-state $\mu$. From the co-state equation in $\mu$

$$
\begin{aligned}
\dot{\mu}(t)= & r \mu(t)-e^{-q(t)}\left\{\lambda^{\prime}(s(t)) V_{2}(s(t))+\lambda(s(t)) V_{2}{ }^{\prime}(s(t))\right\} \\
& -\mu(t) G_{1}{ }^{\prime}(s(t))-\sigma(t) \lambda^{\prime}(s(t))
\end{aligned}
$$

with

$$
\dot{\mu}(t)=-\dot{q}(t) e^{-q(t)} p=-\lambda(s(t)) e^{-q(t)} p
$$

it follows, after eliminating the co-state $\mu$ and multiplying with $e^{q(t)}$, that at each time $t$ 


$$
0=\lambda(s) p+r p-\lambda^{\prime}(s) V_{2}(s)-\lambda(s) V_{2}{ }^{\prime}(s)-p G_{1}{ }^{\prime}(s)-e^{q} \sigma \lambda^{\prime}(s) .
$$

Differentiating this equation with respect to time $t$ for a constant $s$ yields

$$
0=-e^{q(t)} \lambda(s) \sigma(t) \lambda^{\prime}(s)-e^{q(t)} \dot{\sigma}(t) \lambda^{\prime}(s) \Rightarrow \dot{\sigma}(t)=-\lambda(s) \sigma(t)
$$

From this and the co-state equation in $\sigma$

$$
\dot{\sigma}(t)=r \sigma(t)+e^{-q(t)}\left\{p h(t)+\lambda(s(t)) V_{2}(s(t))\right\}
$$

it follows that at each time $t$

$$
(r+\lambda(s)) \sigma=-e^{-q}\left(p h+\lambda(s) V_{2}(s)\right) .
$$

Using this equation to eliminate $e^{q} \sigma$ from (A18) and substituting $h=G_{l}(s)$ finally leads to

$$
G_{1}^{\prime}(s)=r+\lambda(s)\left(1-\frac{V_{2}^{\prime}(s)}{p}\right)+\frac{\lambda^{\prime}(s)}{r+\lambda(s)}\left[G_{1}(s)-\frac{r}{p} V_{2}(s)\right]
$$

This is equation (20) in the main text.

\section{Appendix 2}

In this appendix, we prove a verification theorem for the value function $V I$ constructed in the main part of the article. Recall that the function $V 1$ satisfies the following properties: it is continuous and at least piecewise continuously differentiable. Moreover, there are finitely many points

$$
0=\bar{s}_{1}^{(0)} \leq S_{1}^{(1)} \leq \bar{s}_{1}^{(1)} \leq \cdots \leq s_{1}^{(n)} \leq \bar{s}_{1}^{(n)}=K_{1}
$$

such that

$$
\begin{array}{lll}
V_{1}^{\prime}(s)>p & \text { if } \quad \bar{s}_{1}^{(i-1)}<s<s_{1}^{(i)}, \\
V_{1}^{\prime}(s)=p \quad \text { if } \quad s=s_{1}^{(i)}, \\
V_{1}^{\prime}(s)<p \quad \text { if } \quad s_{1}^{(i)}<s<\bar{s}_{1}^{(i)} .
\end{array}
$$

Only at the points $s=\bar{s}_{1}^{(i)}, i=1, \cdots, n-1$, the function $V_{l}$ may fail to be differentiable. It is continuously differentiable everywhere else, satisfying there the Hamilton-Jacobi-Bellman equation

$$
\max _{0 \leq h \leq h_{m}}\left(p h+\lambda V_{2}-\lambda V_{1}-r V_{1}+V_{1}^{\prime}\left(G_{1}-h\right)\right)=0
$$


Note that the points $s_{1}^{(i)}$ are precisely those points at which the function $f$, defined in the main text, satisfies for $s$ in a neighbourhood of $s_{1}^{(i)}$ that $f(s) \leq 0$ if $s \leq s_{1}^{(i)}$ and $f(s) \geq 0$ if $s \geq s_{1}{ }^{(i)}$. It follows from this that the harvesting rule $h^{*}$ solving the maximisation in (A23) is of the form

$$
\begin{array}{lll}
h^{*}(s)=0 & \text { if } & \bar{s}_{1}^{(i-1)}<s<s_{1}^{(i)}, \\
h^{*}(s)=G\left(s_{1}^{(i)}\right) & \text { if } & s=s_{1}^{(i)}, \\
h^{*}(s)=h_{M} & \text { if } & s_{1}^{(i)}<s<\bar{s}_{1}^{(i)} .
\end{array}
$$

For $s=\bar{s}_{1}^{(i)}$ there are two possibilities:

$$
\begin{aligned}
& h^{*}(s)=\left\{0, h_{M}\right\} \text { if } V_{1} \text { is not differentiable at } s=s_{1}{ }^{(i)}, \\
& h^{*}(s)=G\left(\bar{s}_{1}^{(i)}\right) \quad \text { otherwise. }
\end{aligned}
$$

In the first case, the decision maker is indifferent between two distinct actions; and such a point is called an indifference point (or Skiba point, or DNS(S) point: see Grass et al. (2008)).

We note that if $s$ is the solution of the equation

$$
\dot{s}=G_{1}(s)-h^{*}(s)
$$

with initial condition $s(0)=s_{0}$, then

$$
V_{1}\left(s_{0}\right)=E\left(\int_{0}^{\tau} e^{-r t} p h^{*}(s(t)) d t+e^{-r \tau} V_{2}(s(\tau))\right)
$$

We shall show that $h^{*}(s(t))$ is the optimal harvesting schedule.

Theorem. Let $h=h(t)$ be an admissible harvesting schedule, and let $s=s(t)$ satisfy $s(0)=s_{0}$ and 


$$
\dot{s}(t)=G_{1}(s(t))-h(t) \quad \text { a.e }
$$

Then

$$
V_{1}\left(s_{0}\right) \geq E\left(\int_{0}^{\tau} e^{-r t} p h(t) d t+e^{-r \tau} V_{2}(s(\tau))\right)
$$

and equality obtains if and only if

$$
h(t)=h^{*}(s(t)) \quad \text { a.e. }
$$

Proof. In the following, all harvesting schedules will be assumed to be admissible. We introduce

$$
\Sigma=\left\{s_{1}^{(1)}, \bar{s}_{1}^{(1)}, \cdots, s_{1}^{(n)}, \bar{s}_{1}^{(n)}\right\}
$$

and we make the following fundamental observation. The subset of continuously differentiable harvesting schedules is dense in the set of all schedules, as is the smaller subset of continuously differentiable schedules for which

$$
h^{\prime}(t) \neq 0 \quad \text { whenever } s(t) \in \Sigma
$$

here $s$ is the stock evolution associated to the schedule $h$. The latter property is an easy consequence of Sard's theorem (see e.g. Arnol'd, 1988).

Recall from appendix 1 that

$$
E\left(\int_{0}^{\tau} e^{-r t} p h^{*}(s(t)) d t+e^{-r \tau} V_{2}(s(\tau))\right)=\int_{0}^{\infty} e^{-\int_{0}^{(}(r+\lambda(x)) d x}\left(p h+\lambda V_{2}\right) d t
$$

By the observation above, if there is a harvesting schedule for which (A24) is violated, then 
there is another continuously differentiable schedule $h=h(t)$ satisfying (A25), which also violates (A24). Given this schedule, let $s=s(t)$ be the stock evolution associated to it, and let $h^{*}=h^{*}(s(t))$.

For $s=s(t) \notin \Sigma$, the function $V_{l}$ is differentiable and the following equation obtains:

$$
\frac{d}{d t}\left(e^{-\int_{0}^{(}(r+\lambda(x)) d x} V_{1}\right)=e^{-\int_{0}^{(}(r+\lambda(x)) d x}\left(V_{1}^{\prime}\left(G_{1}-h\right)-(r+\lambda) V_{1}\right)
$$

All arguments $(t)$ and $s(t)$ are suppressed in this equation for readability. There is an increasing sequence

$$
0=t_{0}<t_{1}<\cdots
$$

such that $s=s(t) \notin \Sigma$ if $t \neq t_{j}$, for any j. Note that since the elements of $\Sigma$ are isolated, the sequence $\left\{t_{j}\right\}$ has no accumulation points. Integrating the identity (A27) from $t_{j-1}$ to $t_{j}$ yields

$$
\begin{aligned}
& e^{-\int_{0}^{t_{j}}(r+\lambda(x)) d x} V\left(s\left(t_{j}\right)\right)-e^{-\int_{0}^{j-1}(r+\lambda(x)) d x} V_{1}\left(s\left(t_{j-1}\right)\right) \\
& =\int_{t_{j-1}}^{t_{j}} e^{-\int_{0}^{(}(r+\lambda(x)) d x}\left(V_{1}^{\prime}\left(G_{1}-h\right)-(r+\lambda) V_{1}\right) d t .
\end{aligned}
$$

Summing these expressions over all $j$ yields then

$$
-V_{1}\left(s_{0}\right)=\int_{0}^{\infty} e^{-\int_{0}^{(}(r+\lambda(x)) d x}\left(V_{1}^{\prime}\left(G_{1}-h\right)-(r+\lambda) V_{1}\right) d t .
$$

Adding equations (A26) and (A28) yields

$$
\begin{aligned}
& E\left(\int_{0}^{\tau} e^{-r t} p h(t) d t+e^{-r \tau} V_{2}(s(\tau))\right)-V_{1}\left(s_{0}\right) \\
& \quad=\int_{0}^{\infty} e^{-\int_{0}^{t}(r+\lambda(x)) d x}\left(p h+\lambda V_{2}-\lambda V_{1}-r V_{1}+V_{1}^{\prime}\left(G_{1}-h\right)\right) d t .
\end{aligned}
$$

As $h^{*}$ solves the Hamilton-Jacobi-Bellman equation, subtracting equation (A23) from 
equation (A29) yields

$$
\begin{gathered}
E\left(\int_{0}^{\tau} e^{-r t} p h(t) d t+e^{-r \tau} V_{2}(s(\tau))\right)-V_{1}\left(s_{0}\right) \\
=\sum_{j} \int_{t_{j-1}}^{t_{j}} e^{-\int_{0}^{t}(r+\lambda(x)) d x}\left(p-V_{1}^{\prime}\right)\left(h-h^{*}\right) d t .
\end{gathered}
$$

Now, if $t_{j-1}<t<t_{j}$, then either $V_{1}^{\prime}>p$ and $0=h^{*} \leq h$, or $V_{1}^{\prime}<p$ and $h_{m}=h^{*} \geq h$. In both cases it follows that

$$
\left(p-V_{1}\right)\left(h^{*}-h\right) \leq 0
$$

for almost all $t>0$. Consequently

$$
E\left(\int_{0}^{\tau} e^{-r t} p h(t) d t+e^{-r \tau} V_{2}(s(\tau))\right)-V_{1}\left(s_{0}\right) \leq 0
$$

contradicting the violation of (A24). 